\title{
Charmonium spectral functions from $2+1$ flavour lattice QCD
}

\author{
Szabolcs Borsányi, ${ }^{a}$ Stephan Dürr, ${ }^{a, b}$ Zoltán Fodor, ${ }^{a, b, c}$ Christian Hoelbling, ${ }^{a}$ \\ Sándor D. Katz, ${ }^{c, d}$ Stefan Krieg, ${ }^{a, b}$ Simon Mages, ${ }^{e}$ Dániel Nógrádi,,${ }^{c, d}$ \\ Attila Pásztor, ${ }^{c, d}$ Andreas Schäfer, ${ }^{e}$ Kálmán K. Szabó, ${ }^{a, b}$ Bálint C. Tóth ${ }^{a}$ and \\ Norbert Trombitás ${ }^{c, d}$ \\ ${ }^{a}$ University of Wuppertal, Department of Physics, \\ Wuppertal D-4209\%, Germany \\ ${ }^{b}$ Jülich Supercomputing Center, \\ Jülich D-52425, Germany \\ ${ }^{c}$ Eötvös University, \\ Budapest, H-1117, Hungary \\ ${ }^{d}$ MTA-ELTE Lendület Lattice Gauge Theory Research Group, \\ Budapest, H-1117, Hungary \\ e University of Regensburg, \\ Regensburg D-93053, Germany \\ E-mail: borsanyi@uni-wuppertal.de, durr@itp.unibe.ch, \\ fodor@physik.uni-wuppertal.de, hch@uni-wuppertal.de, \\ katz@bodri.elte.hu, s.krieg@fz-juelich.de, \\ simon-wolfgang . mages@physik. uni-regensburg.de, \\ nogradi@bodri.elte.hu, apasztor@bodri.elte.hu, \\ andreas.schaefer@physik.uni-regensburg.de, szaboka@general.elte.hu, \\ tothbalint@szofi.elte.hu, trombitas@bodri.elte.hu
}

ABSTRACT: Finite temperature charmonium spectral functions in the pseudoscalar and vector channels are studied in lattice QCD with $2+1$ flavours of dynamical Wilson quarks, on fine isotropic lattices (with a lattice spacing of $0.057 \mathrm{fm}$ ), with a non-physical pion mass of $m_{\pi} \approx 545 \mathrm{MeV}$. The highest temperature studied is approximately $1.4 T_{c}$. Up to this temperature no significant variation of the spectral function is seen in the pseudoscalar channel. The vector channel shows some temperature dependence, which seems to be consistent with a temperature dependent low frequency peak related to heavy quark transport, plus a temperature independent term at $\omega>0$. These results are in accord with previous calculations using the quenched approximation.

KEYworDS: Lattice QCD, Phase Diagram of QCD

ARXIV EPRINT: 1401.5940 


\section{Contents}

1 Introduction $\quad 1$

1.1 Motivation 1

1.2 Mesonic spectral functions 2

1.3 The Maximum Entropy Method 3

1.4 Lattice configurations 5

1.4.1 Charm mass tuning 5

1.4.2 Approximation of the "critical temperature" 6

2 Results $\quad 6$

2.1 Zero temperature analysis 6

$\begin{array}{lll}2.2 & \text { MEM reconstructed spectral functions } & 7\end{array}$

$\begin{array}{lll}2.2 .1 & \text { Pseudoscalar channel } & 7\end{array}$

$\begin{array}{ll}2.2 .2 \text { Vector channel } & 7\end{array}$

$\begin{array}{ll}2.3 \text { The ratio } G / G_{\text {rec }} & 10\end{array}$

3 Summary and outlook $\quad 11$

$\begin{array}{ll}\text { A MEM details } & \mathbf{1 3}\end{array}$

$\begin{array}{ll}\text { A.1 Numerical implementation } & 13\end{array}$

$\begin{array}{lll}\text { A.2 Error analysis } & 14\end{array}$

\section{Introduction}

\subsection{Motivation}

As the early universe expanded after the Big Bang, a transition occurred at $T \approx 150 \mathrm{MeV}$, from the so called quark-gluon plasma to a confined phase with hadrons. The nature of this transition affects our understanding of the history of the universe, see e.g. [1].

Extensive experimental work is currently done at both RHIC and LHC, trying to understand the QCD aspects of the early universe with heavy ion collisions. Both for the cosmological transition and for these heavy ion experiments, the densities are quite small, and so the baryonic chemical pontentials are much less than the typical hadron masses. Therefore a $\mu=0$ calculation is a good approximation for both cases.

The $\mu=0$ transition can be studied using lattice gauge theory. There are various results using different fermion regularizations. Within the staggered formalism there are continuum extrapolated results at physical quark masses, such as the nature of the transition, the transition temperature, equation of state and fluctuations [2-8]. There are also results with the computationally more expensive, but conceptually cleaner Wilson fermions [9-15], but so far only at non-physical masses, here however, continuum results 
are also available [16]. In this paper, we use Wilson fermions, since spectroscopy is easier to handle [17], and we will need to measure correlators at finite temperatures. We use a fine lattice spacing of $a=0.057 \mathrm{fm}$, but continuum extrapolation does not seem feasible at this point.

The charmonium systems have been under heavy investigation, since the suppression of $J / \Psi$ is regarded as an important experimental signal for the formation of the plasma state [18]. Charmonium states are expected to dissolve somewhat above the transition temperature, because of the screening of the inter-quark potential and collisions with the hot medium. In this paper, we investigate the in medium properties of the $J / \Psi$ and $\eta_{c}$ mesons from a lattice QCD perspective.

\subsection{Mesonic spectral functions}

The spectral function (SF) of a correlator of self-adjoint operators is the imaginary part of the Fourier-transform of the real time retarded correlator [19].

$$
\begin{aligned}
A_{H}(\omega) & =\frac{1}{\pi} \Im D_{H}^{R}(\omega, \vec{p})=\frac{1}{2 \pi}\left(D_{H}^{>}(\omega, \vec{p})-D_{H}^{<}(\omega, \vec{p})\right), \\
D_{H}^{>(<)}(t, \vec{x}) & =\int \frac{d^{4} p}{(2 \pi)^{4}} e^{i p x} D_{H}^{>(<)}(\omega, \vec{p}), \quad \text { where } \\
D_{H}^{>}(t, \vec{x}) & =\left\langle J_{H}(t, \vec{x}) J_{H}(0, \overrightarrow{0})\right\rangle, \quad t>0 \text { and } \\
D_{H}^{<}(t, \vec{x}) & =\left\langle J_{H}(0, \overrightarrow{0}) J_{H}(t, \vec{x})\right\rangle, \quad t>0 .
\end{aligned}
$$

In this article, we will deal with correlators between mesonic currents, and the corresponding mesonic SFs. These operators schematically look like

$$
J_{H}(t, \vec{x})=\bar{q}(\vec{x}, t) \Gamma_{H} q(\vec{x}, t),
$$

where $q$ is the quark field and $\Gamma_{H}=\gamma_{5}, \gamma_{i}$ for the pseudoscalar and vector channels respectively.

It can be shown, that the SF is related to the Euclidean correlator - calculable on the lattice - by an integral transform

$$
G(\tau, \vec{p})=\int_{0}^{\infty} d \omega A(\omega, \vec{p}) K(\omega, \tau),
$$

where we dropped the subscript $\mathrm{H}$,

$$
K(\omega, \tau)=\frac{\cosh (\omega(\tau-1 / 2 T))}{\sinh (\omega / 2 T)}
$$

is the integral kernel, and the Euclidean correlator (at zero chemical potential) is:

$$
G(\tau, \vec{p})=D_{H}^{>}(-i \tau, \vec{p})=\int d^{3} x e^{i \vec{p} \vec{x}}\left\langle T_{\tau} J_{H}(-i \tau, \vec{x}) J_{H}(0, \overrightarrow{0})\right\rangle .
$$

Knowledge of these SFs is of great importance. Inserting a complete set of states and using the so-called Kubo-Martin-Schwinger condition, one can show that:

$$
A(\omega, \vec{p})=\frac{(2 \pi)^{2}}{Z} \sum_{m, n}\left(e^{-E_{n} / T}-e^{-E_{m} / T}\right)\left|\left\langle n\left|J_{H}(0)\right| m\right\rangle\right|^{2} \delta^{(4)}\left(p_{\mu}-k_{\mu}^{n}+k_{\mu}^{m}\right),
$$


where $k_{i}=\left(E_{i}, \vec{k}_{i}\right)$ and $p=(\omega, \vec{p})$. In this sum, a stable particle gives a $\delta$ like peak [19], while an unstable particle in matter gives a smeared peak. Also, an important result of linear response theory is the Kubo-formula, which states that the transport coefficients are related to the zero frequency limit of $A(\omega) / \omega$. In particular, the heavy quark diffusion constant $\mathrm{D}$ is related to the vector spectral function:

$$
D=\frac{1}{6 \chi} \lim _{\omega \rightarrow 0} \sum_{i=1}^{3} \frac{\rho_{i i}(\omega, T)}{\omega},
$$

where $\chi$ is the (heavy) quark number susceptibility and $\rho_{i i}$ is the spectral function corresponding to the vector channel. If the transport coefficient is non vanishing, we expect some finite value of $\rho / \omega$ for small $\omega$. This implies the presence of a transport peak. We will investigate the anticipated melting of the heavy meson states $J / \Psi$ and $\eta_{c}$ in the quark gluon plasma, which is supposed to happen somewhat above the transition temperature [18]. As one increases the temperature, the width of a given peak increases and at sufficiently high temperatures, the contribution from the meson state in the SF may be sufficiently broad so that it is not very meaningful to speak of it as a well defined state any more. If the peak corresponding to the given states disappears from the SF in this way, we can say it melted.

In the following, we will only be dealing with SFs at zero spatial momentum $A(\omega, \overrightarrow{0})=$ $A(\omega)$, but we point out that the analysis of the non-zero spatial momentum SFs would go the same way, except one would need to start from non-zero spatial momentum correlators.

\subsection{The Maximum Entropy Method}

To get the SFs from a lattice study one has to invert equation (1.6). This inversion however is ill-defined, since the typical number of frequencies for which one wants to reconstruct the $\mathrm{SF}$ is higher than the number of data points. In this case a $\chi^{2}$ fit on the shape of the SF discretized to $N_{\omega}$ points is degenerate. One has to regularize the problem in some way. ${ }^{1}$

The determination of hadronic SFs via the Maximum Entropy Method (MEM) was first suggested in [20]. This is a regularization that has some justification from Bayesian probability theory. One has to maximize:

$$
Q=\alpha S-\frac{1}{2} \chi^{2}
$$

where as usual

$$
\chi^{2}=\sum_{i, j=1}^{N_{\text {data }}}\left(G_{i}^{\mathrm{fit}}-G_{i}^{\mathrm{data}}\right) C_{i j}^{-1}\left(G_{j}^{\mathrm{fit}}-G_{j}^{\mathrm{data}}\right),
$$

with $C_{i j}$ being the covariance matrix of the data (in Euclidean time), and the ShannonJaynes entropy ${ }^{2}$ is

$$
S=\int d \omega\left(A(\omega)-m(\omega)-A(\omega) \log \left(\frac{A(\omega)}{m(\omega)}\right)\right)
$$

\footnotetext{
${ }^{1}$ Note that the number of data points vs. parameters is not the only problem when trying to reconstruct SFs. Even if one has a large number of data points (e.g. on time anisotropic lattices), the Euclidean correlator is rather insensitive to fine details of the SF. Therefore the inversion introduces large uncertainties [21].

${ }^{2}$ This is the generalization of the Shannon entropy to continuous probability distributions, it is the negative of the Kulback-Leibler divergence.
} 
where the so called prior function $m(\omega)$ is supposed to summarize our prior knowledge on the shape of the SF (such as the leading perturbation theory behaviour). It can then be shown that the maximum of $Q$ lies in an $N_{\text {data }}$ dimensional subspace of the $N_{\omega}$ dimensional space of possible $A\left(\omega_{i}\right)$ vectors, that can be parametrized as:

$$
A(\omega)=m(\omega) \exp \left(\sum_{i=1}^{N_{\mathrm{data}}} s_{i} f_{i}(\omega)\right) .
$$

The most widely used choice for basis functions involves a Singular Value Decomposition and is called the Bryan method. It was introduced in [22]. The particular choice of the basis for the subspace we use is $f_{i}(\omega)=K\left(\omega, \tau_{i}\right)$ and was introduced by ref. [23]. In our experience this proved to be numerically more stable than the former one. In this case the maximization of $\mathrm{Q}$ is equivalent to the minimization of

$$
U=\frac{\alpha}{2} \sum_{i, j=1}^{N_{\text {data }}} s_{i} C_{i j} s_{j}+\int_{0}^{\omega_{\max }} \mathrm{d} \omega \mathrm{A}(\omega)-\sum_{\mathrm{i}=1}^{\mathrm{N}_{\text {data }}} \mathrm{G}_{\mathrm{i}}^{\text {data }} \mathrm{S}_{\mathrm{i}} .
$$

One can see from equation (1.14) that the shape of the subspace is strongly dependent on the choice of the prior function. This is the source of a systematic uncertainty, that has to be considered.

After equation (1.15) is minimized at a given value of $\alpha$, and the optimal $A_{\alpha}$ is obtained, the regularization parameter $\alpha$ has to be averaged over. Using Bayes' theorem, one can show that the conditional probability of $\alpha$ having a specific value, given the data and the prior function is [20]:

$$
P[\alpha \mid D, m] \propto \exp \left(\frac{1}{2} \sum_{k} \log \frac{\alpha}{\alpha+\lambda_{k}}+\alpha S-\frac{1}{2} \chi^{2}\right),
$$

where the $\lambda$-s are the eigenvalues of the matrix $\Lambda_{l, l^{\prime}}=\frac{1}{2}\left(\sqrt{A_{l}} \frac{\partial^{2}\left(\chi^{2}\right)}{\partial A_{l} \partial A_{l^{\prime}}} \sqrt{A_{l^{\prime}}}\right)_{A=A_{\alpha}}$. The efficient marginalization of $\alpha$ involves a trick using Sylvester's determinant theorem, that can be found in the appendix of [25].

We also mention, that we use a modified version of the kernel and the spectral functions for the reconstruction $[26,27]$ :

$$
\hat{K}(\tau, \omega)=\tanh (\omega / 2) K(\tau, \omega),
$$

and

$$
\hat{A}(\omega)=\operatorname{coth}(\omega / 2) A(\omega) .
$$

This "cures" the low frequency divergent $1 / \omega$ behaviour of the kernel, without spoiling the high $\omega$ behaviour. In the rest of the paper, we will only be dealing with Euclidean time, and we will simply denote it with $t$ (changing the notation from $\tau$ ).

Lattice studies of charmonium SFs using the MEM have been carried out on numerous occasions $([23,28-35])$, but so far not in $2+1$ flavour QCD. A recent, detailed study 
of charmonium SFs in quenched QCD can be found in [34]. Results regarding spectral functions with 2 flavours of dynamical quarks can be found in refs. [31, 35]. A recent study of electric conductivity using $2+1$ flavours of anisotropic Wilson fermions can be found in [36]. Another interesting application of the spectral function reconstruction is the study of the melting bottonium states in the context of Non-relativistic QCD [37]. Some details about the numerical implementation and the error analysis can be found in appendix A.

\subsection{Lattice configurations}

We use the same lattice configurations as in [16]. The gauge action used for the calculations was the Symanzik tree level improved gauge action [38, 39]

$$
S_{G}^{\text {Sym }}=\beta\left[\frac{c_{0}}{3} \sum_{\text {plaq }} \operatorname{Re} \operatorname{Tr}\left(1-U_{\text {plaq }}\right)+\frac{c_{1}}{3} \sum_{\text {rect }} \operatorname{Re} \operatorname{Tr}\left(1-U_{\text {rect }}\right)\right],
$$

with the parameters $c_{0}=5 / 3$ and $c_{1}=-1 / 12$. The action for the fermionic sector was the clover improved [40] Wilson action

$$
S_{F}^{\mathrm{SW}}=S_{F}^{\mathrm{W}}-\frac{c_{\mathrm{SW}}}{4} \sum_{x} \sum_{\mu, \nu} \bar{\psi}_{x} \sigma_{\mu \nu} F_{\mu \nu, x} \psi_{x}
$$

where $S_{F}^{\mathrm{W}}$ is the Wilson fermion action. Six steps of stout smearing [41] with smearing parameter $\varrho=0.11$ were used. The clover coefficient was set to its tree level value, $c_{\mathrm{SW}}=1.0$, which, for this type of smeared fermions, essentially leads to an $\mathcal{O}(a)$ improved action [42] with improved chiral properties [43]. The same action was first used in ref. [44] where the excellent scaling properties of hadron masses was observed. The full hadron spectrum using this action was determined in ref. [17].

The bare masses of the $u$ and $d$ quarks were taken to be degenerate, therefore the configurations were generated using an $N_{f}=2+1$ flavor algorithm. The $u$ and $d$ quarks were implemented via the Hybrid Monte Carlo (HMC) algorithm [45], whereas the strange quark was implemented using the Rational Hybrid Monte Carlo (RHMC) algorithm [46]. In order to speed up the molecular dynamics calculations, the Sexton-Weingarten multiple time-scale integration scheme [47] combined with the Omelyan integrator [48] was employed. When all four extents of the lattice were even, the usage of even-odd preconditioning [49] gave an additional speed up factor of 2 .

From the study in [16], we only used the finest lattices, with gauge coupling $\beta=3.85$, corresponding to a lattice spacing of $a=0.057(1) \mathrm{fm}$. The bare light quark masses where choosen to be $a m_{u d}=-0.00336$ and $a m_{s}=0.0050$, which, when fixing the scale with a physical $\Omega$ baryon mass, corresponds to a pion mass $m_{\pi} \approx 545 \mathrm{MeV}$. A summary of the lattices used can be found in table 1.

\subsubsection{Charm mass tuning}

From ref. [50] the ratio $m_{c} / m_{s}=11.85$. Since with Wilson fermions, there is an additive renormalization, it is not possible to use this ratio directly in setting the charm mass. However, we know that for $u d$ and $s$ the masses used in the simulation correspond to a 


\begin{tabular}{|cccccccc|}
\hline$a[\mathrm{fm}]$ & $a m_{u d}$ & $a m_{s}$ & $m_{\pi}$ & $N_{s}$ & $N_{t}$ & $T=\frac{1}{N_{t} a}$ & $\#$ confs. \\
\hline $0.057(1)$ & -0.00336 & 0.0050 & $545 \mathrm{MeV}$ & 64 & 28 & $123 \mathrm{MeV}$ & 151 \\
$0.057(1)$ & -0.00336 & 0.0050 & $545 \mathrm{MeV}$ & 64 & 20 & $173 \mathrm{MeV}$ & 95 \\
$0.057(1)$ & -0.00336 & 0.0050 & $545 \mathrm{MeV}$ & 64 & 18 & $192 \mathrm{MeV}$ & 328 \\
$0.057(1)$ & -0.00336 & 0.0050 & $545 \mathrm{MeV}$ & 64 & 16 & $216 \mathrm{MeV}$ & 254 \\
$0.057(1)$ & -0.00336 & 0.0050 & $545 \mathrm{MeV}$ & 64 & 14 & $247 \mathrm{MeV}$ & 411 \\
$0.057(1)$ & -0.00336 & 0.0050 & $545 \mathrm{MeV}$ & 64 & 12 & $288 \mathrm{MeV}$ & 300 \\
\hline
\end{tabular}

Table 1. The different lattices used in the study. The configurations are separated by 5 trajectories. We have explicitly checked, that none of the covariance matrices $C_{i j}$ of the data we have display pathological spectra.

\begin{tabular}{|lllllll|}
\hline$J^{P}$ & $m_{i}$ & name & ma & $m a / m_{D_{s}^{*}} a$ & $m_{\exp }[\mathrm{MeV}]$ & $m_{\text {exp }} / m_{D_{s}^{*}}$ \\
\hline $0^{-}$ & $m_{s}, m_{c}$ & $D_{s}$ & $0.54(1)$ & $0.95(2)$ & 1968.4 & 0.932 \\
$0^{-}$ & $m_{c}, m_{c}$ & $\eta_{c}$ & $0.8192(7)$ & $1.437(4)$ & 2981.0 & 1.411 \\
$1^{-}$ & $m_{s}, m_{c}$ & $D_{s}^{*}$ & $0.570(1)$ & 1 & 2112.3 & 1 \\
$1^{-}$ & $m_{c}, m_{c}$ & $J / \Psi$ & $0.8388(8)$ & $1.472(2)$ & 3096.916 & 1.466 \\
$3 / 2^{+}$ & $3 m_{s}$ & $\Omega$ & $0.478(8)$ & $0.84(2)$ & 1672.45 & 0.791 \\
\hline
\end{tabular}

Table 2. The different hadron masses, obtained by fitting $A \cosh \left(m a\left(t-N_{t} / 2\right)\right)$ to smeared correlators (in case of mesons, sinh in case of baryons) on $64^{4}$ lattices.

mass ratio of $1.5[51,52]$, from this we get $\left(m_{c}-m_{s}\right) /\left(m_{s}-m_{u d}\right)=35.55$ which gives the estimate for the charm mass that was used. To check if this is approximately the correct charm mass, we checked the masses of the different mesons states containing $\mathrm{s}$ and c quarks, and they were indeed in the right ballpark. See table 2.

\subsubsection{Approximation of the "critical temperature"}

Since the simulation is done at a non-physical pion mass, instead of giving the temperature values in $\mathrm{MeV}$, it is probably more appropriate to give temperatures in $T_{c}$ units. Since with dynamical light quarks there is no phase transition, the transition is a cross-over, there is a multitude of possible definitions of $T_{c}$. We will use the temperature value where the tree-level improved strange quark susceptibility equals 0.5 . One can obtain an estimate of this temperture from figure 2 of ref. [16], where the continuum extrapolated strange quark susceptibility is plotted with systematic and statistical errors. The transition temperature is $T / m_{\Omega}=0.110(2)$.

\section{Results}

\subsection{Zero temperature analysis}

$\mathrm{S}$ ince the temperature is $T=1 /\left(N_{t} a\right)$, as the temperature increases we have less and less data points for our reconstruction of the SFs. That means that the reliability of the method decreases with increasing temperature. So we need an estimate of the highest temperature, 


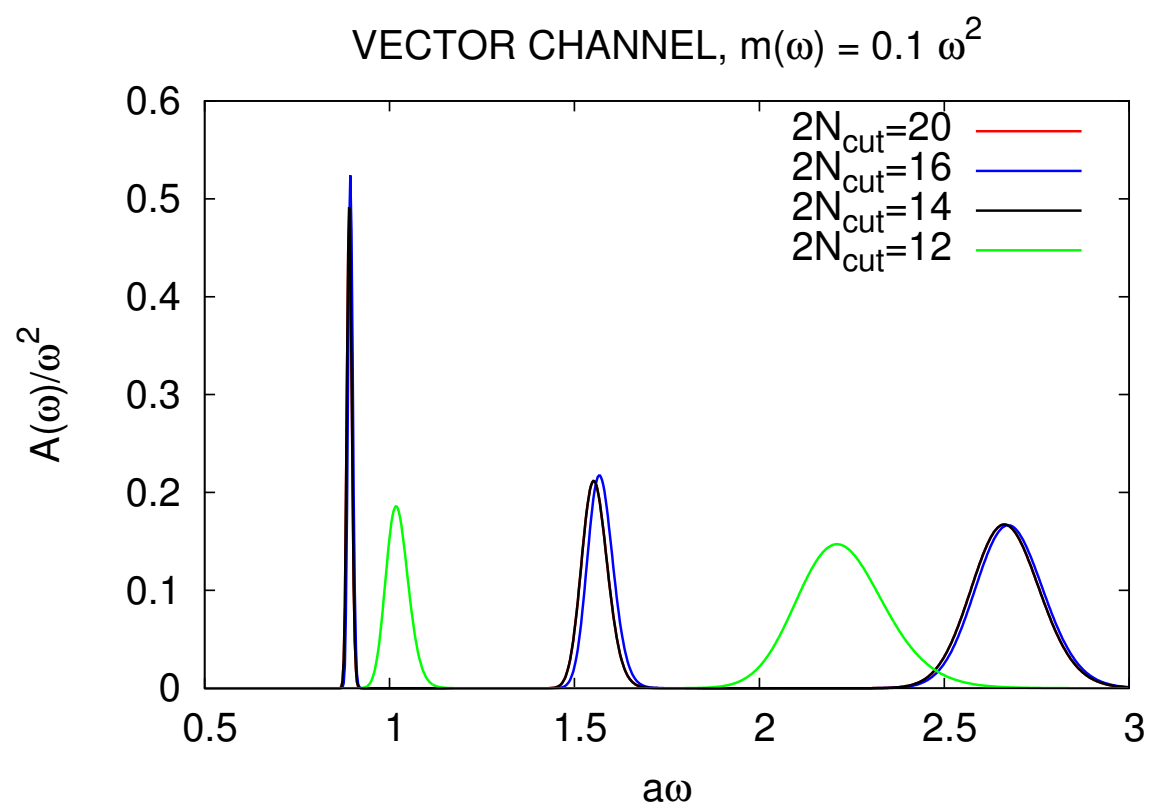

Figure 1. The results of dropping points from the zero temperature reconstruction. We dropped points starting from $t=0$, using only the points closest to $t=N_{t} / 2 . N_{\text {cut }}$ is the number of points used for reconstruction. For $2 N_{\text {cut }} \leq 12$ one can no longer reconstruct the first peak.

where the MEM results are still likely to be trusted. To get such an estimate, we drop points from the lowest temperature correlators and do a MEM reconstruction with these limited number of points. We say that the reconstruction is no longer reliable when we can not reconstruct the first peak. The results are illustrated in figure 1. As we can see, $N_{t}=12$ is already not reliable, meaning that the highest temperature we can study with direct MEM reconstruction is corresponding to $N_{t}=14 .^{3}$

\subsection{MEM reconstructed spectral functions}

\subsubsection{Pseudoscalar channel}

Reconstructions of the pseudoscalar (PS) SF with different prior functions can been seen in figure 2. Reconstruction of the PS spectral function with the same prior functions at different temperatures can be seen in figure 3. Looking at these pictures together one can draw the intuitive conclusion that the difference in the SFs at various temperatures is smaller than the reconstruction error coming from the variation of the reconstruction with different prior functions. So as far as our analysis can tell, the PS SF is temperature independent in the given range. This is further confirmed by figure 4 , which shows a full error analyis of the peak position.

\subsubsection{Vector channel}

The situation is a little bit more complicated in the vector channel. Reconstructions of the vector SF with different prior functions can been seen in figure 2. Reconstruction of the

\footnotetext{
${ }^{3}$ Of course, this is not an absolute criterion.
} 

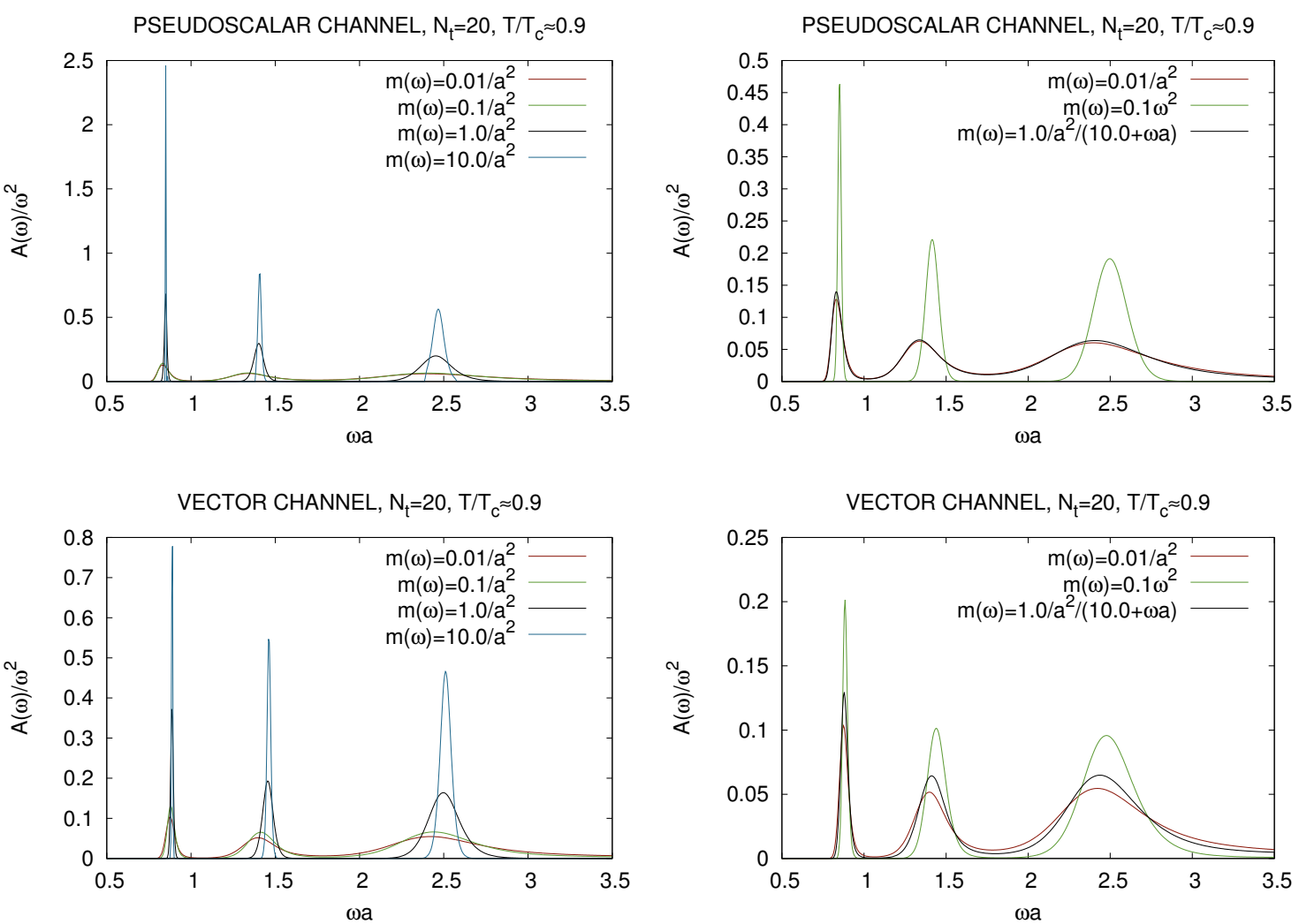

Figure 2. The sensitivity of the reconstruction on the prior function. Note that the widths of the peaks have large systematic errors, as can be seen from the reconstruction with different prior functions.
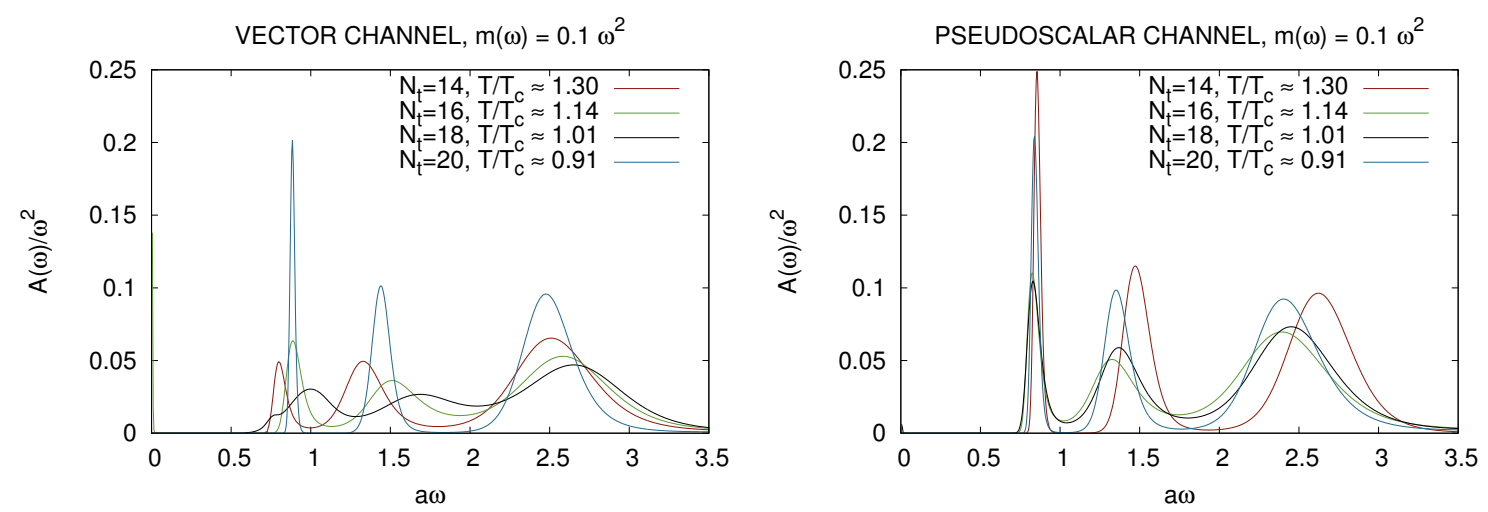

Figure 3. The temperature dependence of the reconstucted spectral functions.

vector SF with the same prior functions at different temperatures can be seen in figure 3 . In this last plot the highest temperature seems to differ from the other temperatures. The first peak appears to go down to lower temperatures. Due to some properties of the analysis (i.e. possible merging of adjacent peaks and problems with the resolution of the transport peak) using MEM alone one cannot draw any firm conclusions about the nature of the change in the SF - at least at the current level of statistical errors. At $N_{t}=16$ the MEM 

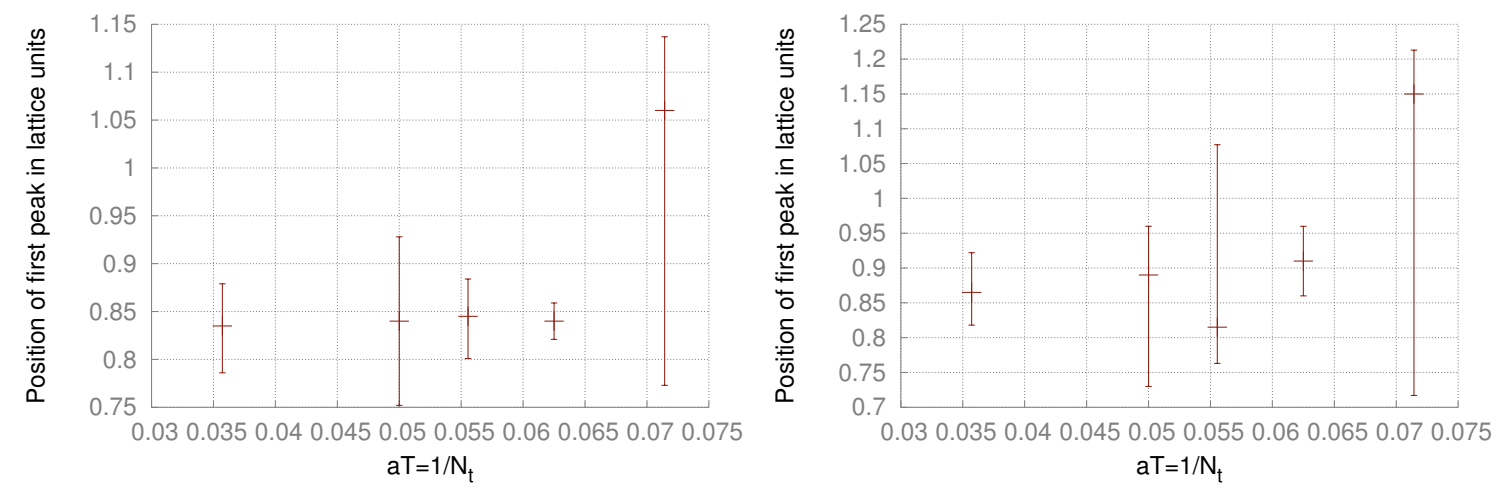

Figure 4. The position of the first pseudoscalar(left) and vector(right) peak as a function of temperature. The big error bars at the highest temperature, corresponding to $N_{t}=14$ come from the fact that with so few data points, the MEM procedure merges the first two peaks with some prior functions. Errorbars include both systematic and statistical errors.
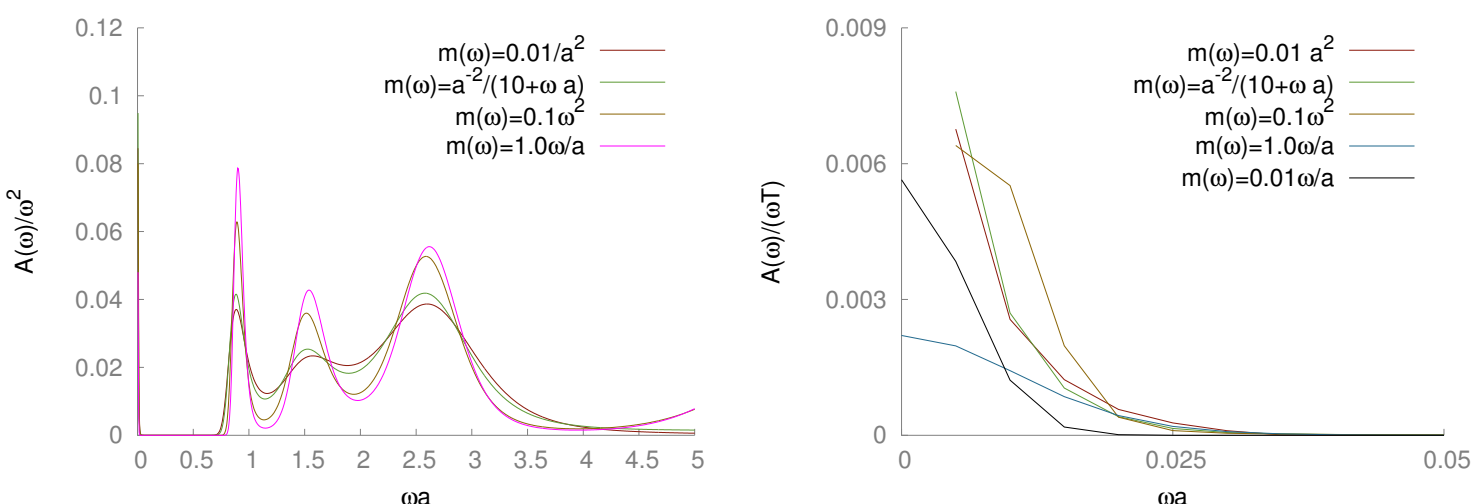

Figure 5. The MEM reconstructed vector $\mathrm{SF}$ at $N_{t}=16$ in the vector channel. Notice the indication of a transport peak in the MEM analysis. With the prior functions $A(\omega)=m_{0} a^{-2}$ or $\frac{a^{-2}}{m_{0}+\omega a}$ the zero frequency limit of $A(\omega) / \omega$ is always infinity, with $A(\omega)=m_{0} \omega^{2}$ it is always zero. To actually get a value for the transport coefficients, we need to use the prior function $A(\omega)=m_{0} \omega a^{-1}$. This way we can get an estimate, but as the results show, it has a big systematic uncertainty. As we will stress in the analysis of the "reconstructed" correlators, the data are not really sensitive to the zero frequency intercept, only the area of the transport peak.

reconstruction picks up a transport related low frequency peak (see figure 5). However at $N_{t}=14$ the MEM is already not reliable in the vector channel. From mock data analysis, we observed that MEM can merge two close lying peaks to one peak between the two real peaks. We suspect that this is what is happening at $N_{t}=14$. The $N_{t}=14$ reconstruction also picks up a transport peak with some prior functions, but here we don't see three $\omega>0$ peaks together with a transport peak. The first peak is always merged with the transport peak, or the second peak. This peak merging property makes the error bars on the peak position at this temperature so big. Figure 4 shows a full error analyis of the peak position. The actual physical picture will be clarified in the next point of our analysis. 


\subsection{The ratio $G / G_{\text {rec }}$}

An alternative aproach to study spectral functions was suggested in [23]. The ratio:

$$
\frac{G(t, T)}{G_{\mathrm{rec}}(t, T)}=\frac{G(t, T)}{\int A\left(\omega, T_{\mathrm{ref}}\right) K(\omega, t, T) \mathrm{d} \omega}
$$

has a few advantages:

- MEM reconstruction is only needed at $T_{\text {ref }}$, where we have the most data points, and so a more reliable reconstruction. We use $N_{t}=28$ as reference temperature.

- We can calculate this ratio even at high temperatures, where the MEM reconstruction is already unreliable.

- If the spectral function is temperature independent, then the trivial temperature dependence of the correlators, coming from the integral kernel will drop out, and the ratio will be $G / G_{\text {rec }}=1$.

It is also useful to study the same ratio with mid-point subtracted correlators [24].

$$
\begin{aligned}
\frac{G^{-}}{G_{\text {rec }}^{-}} & =\frac{G(t, T)-G\left(N_{t} / 2, T\right)}{G_{\text {rec }}(t, T)-G_{\text {rec }}\left(N_{t} / 2, T\right)} \\
& =\frac{G(t, T)-G\left(N_{t} / 2, T\right)}{\int A\left(\omega, T_{\text {ref }}\right)\left[K(\omega, t, T)-K\left(\omega, N_{t} / 2, T\right)\right] \mathrm{d} \omega}
\end{aligned}
$$

This way, one can drop the zero-mode (constant) contribution to the correlators. These have to do with transport coefficients, or other low frequency $(\omega \ll T)$ features of the spectral functions.

If the ratio of $G / G_{\text {rec }}$ is different from one, but the ratio with the middle-point substracted correlators is not, that means that the temperature dependence of the SFs should be well described by just a zero-mode contribution $f(T) \cdot \omega \delta\left(\omega-0^{+}\right)$. The results of such an analysis can be seen in figures 6 and 7. As one can see, the results in the pseudoscalar channel are consistent with a temperature independent SF, while the results in the vector channel show a temperture dependent zero mode/low frequency contribution in the SF. We can also try to extract the zero mode contribution itself by considering the difference $G-G_{\text {rec }}$. This is only plotted in the vector channel, in figure 8 (in the pseudovector channel it is always consistent with zero). The difference has big errors, but on the two highest temperatures it is non zero within $1 \sigma$. At every temperature it is consistent with a time separation independent constant. With the ansatz $A(\omega, T)=f(T) \omega \delta\left(\omega-0^{+}\right)+A\left(\omega, T_{0}\right)$ we get $f(T) T \approx(3 \pm 1.5) \cdot 10^{-5}$ at $1.5 T_{c}$ in lattice units. ${ }^{4}$ This ansatz, taken strictly, would imply a diverging diffusion constant. However, the data do not restrict the shape of the transport peak, they are only sensitive to the area. By using this ansatz, we do not mean to say that the diffusion constant diverges, we simply extract that area of the transport

\footnotetext{
${ }^{4}$ Since we are not using the conserved current on the lattice, but a local current, this will have a finite, lattice spacing dependent renormalization constant of $\mathcal{O}(1)$. We neglect this fact, since we don't do a continuum limit, and the renormalization is temperature independent.
} 

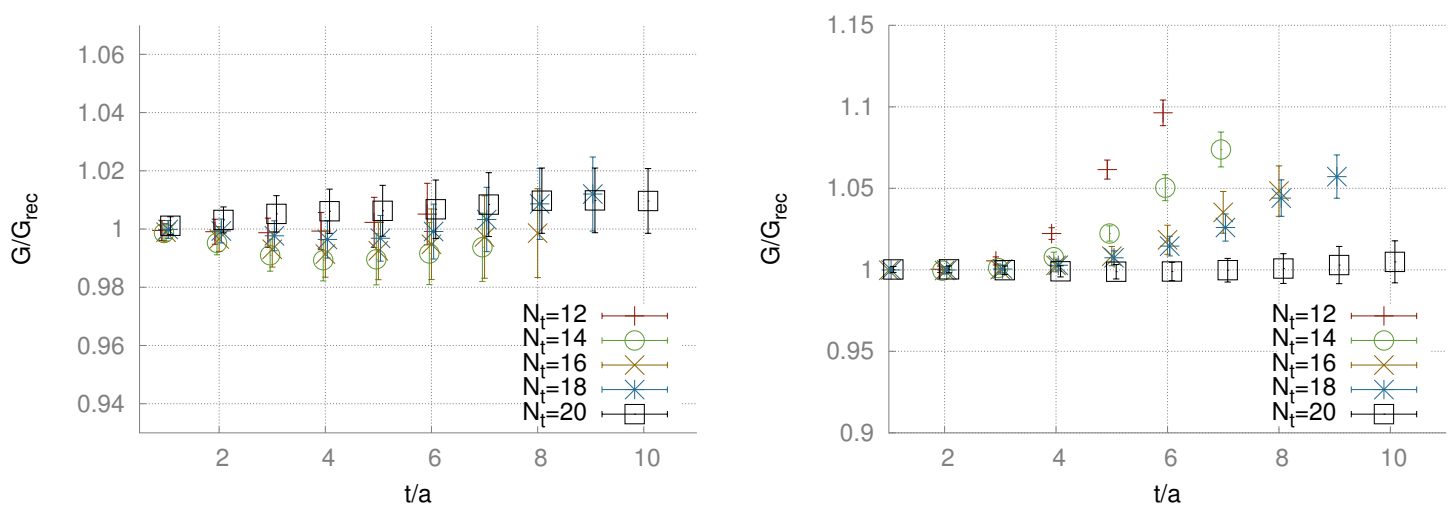

Figure 6. The ratio $G / G_{\text {rec }}$ in the pseudoscalar(left) and vector(right) channels. Errorbars include both systematic and statistical errors.
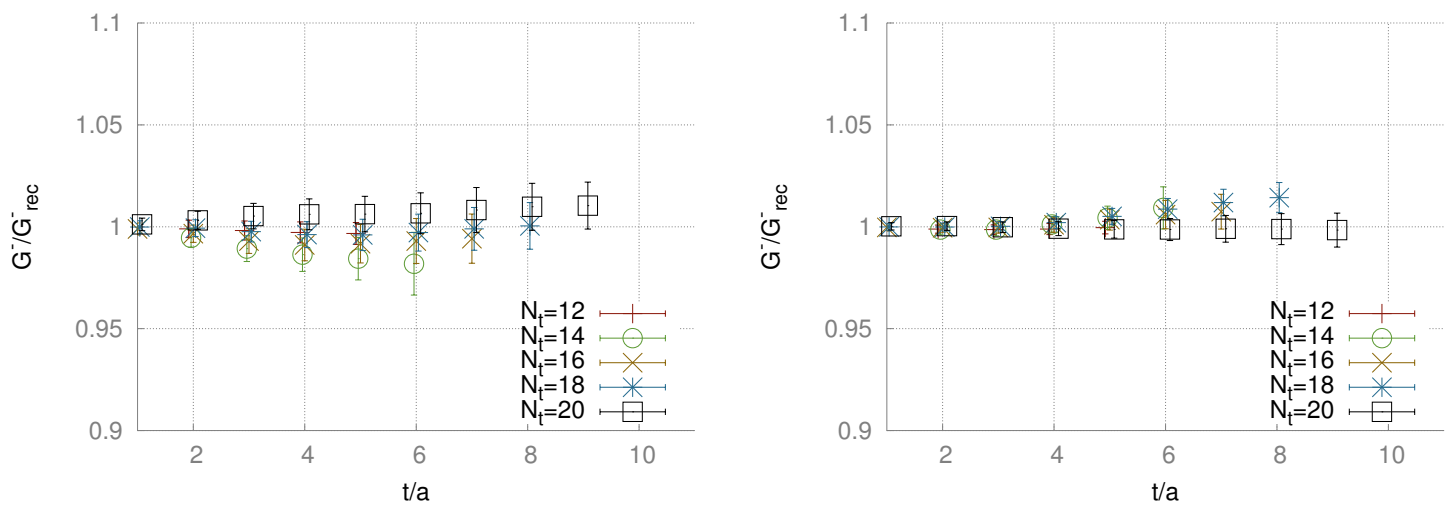

Figure 7. The ratio $G^{-} / G_{\text {rec }}^{-}$in the pseudoscalar(left) and vector(right) channels. Errorbars include both systematic and statistical errors.

peak. To get a diffusion constant additional information is needed. (The width or the height of the peak, which is too narrow to resolve at this point.) The survival of $J / \Psi$ up to such high temperatures is consistent with previous results in quenched and 2 flavour QCD (see eg. [31, 33, 34]).

\section{$3 \quad$ Summary and outlook}

We have performed a lattice study of charmonium spectral functions with $2+1$ dynamical Wilson quarks. The MEM reconstruction of the spectral functions is hampered by the limited number of data points at higher temperatures, so the highest temperature we used for MEM reconstruction was approximately $1.3 T_{c}$. The PS spectral functions did not show any noticable temperature variation. The $\mathrm{V}$ spectral functions showed a temperature variation, which, according to the analysis of the ratio $G / G_{\text {rec }}$ is consistent with a temperature dependent zero mode, and a temperature independent non-zero part in the SFs. In conclusion, we can say that we observed no melting of the $\eta_{c}$ and $J / \Psi$ mesons up to temperature of $1.5 T_{c}$, and we observe no variations in the spectral functions of $\eta_{c}$ whatsoever. The varia- 


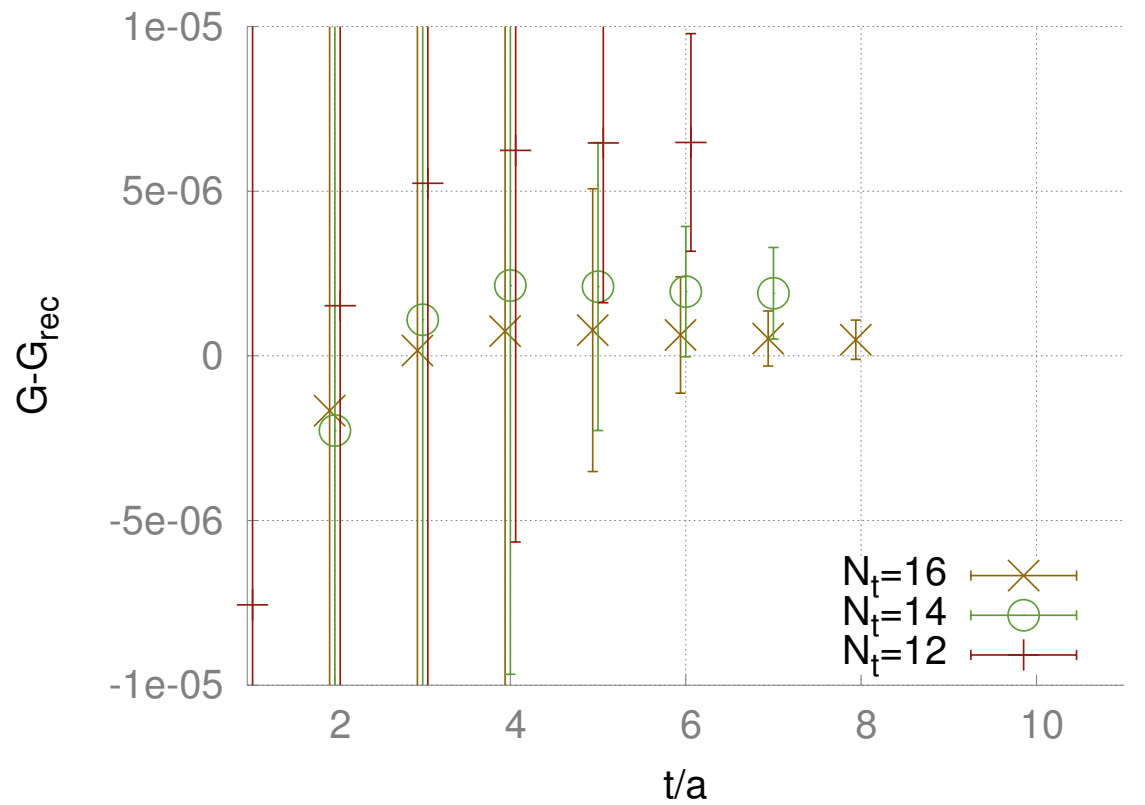

Figure 8. The difference $G-G_{\text {rec }}$ in the vector channel at the 3 highest temperatures. If we assume the ansatz $A(\omega, T)=f(T) \omega \delta\left(\omega-0^{+}\right)+A\left(\omega, T_{0}\right)$ the difference is equal to $f(T) T / 2$. Errorbars include both systematic and statistical errors.

tions in vector SF can be well described by the ansatz $A(\omega, T)=f(T) \omega \delta\left(\omega-0^{+}\right)+A\left(\omega, T_{0}\right)$ with $f(T) T \approx(3 \pm 1.5) \cdot 10^{-5}$ at $1.5 T_{c}$ in lattice units, giving the area of the transport peak.

Mock data analysis shows that the errors of the data points are more important than the number of data points itself (see e.g. [20]), so anisotropic lattices are not expected to substantially increase the accuracy. However because of the increased number of data point at higher temperatures, the reconstruction is likely to be reliable at higher temperatures than with isotropic lattices. Anisotropy tuning with dynamical fermions is a difficult task, but it can somewhat be made easier by the method described in [53]. This will be the main direction for future studies.

\section{Acknowledgments}

We thank P. Petreczky and A. Jakovác for useful discussions.

Computations were carried out on GPU clusters [54] at the Universities of Wuppertal and Budapest as well as on supercomputers in Forschungszentrum Juelich.

This work was supported by the EU Framework Programme 7 grant (FP7/20072013)/ERC No 208740, by the Deutsche Forschungsgemeinschaft grants FO 502/2, SFB-TR 55 and by Hungarian Scientific Research Fund grant OTKA-NF-104034. 

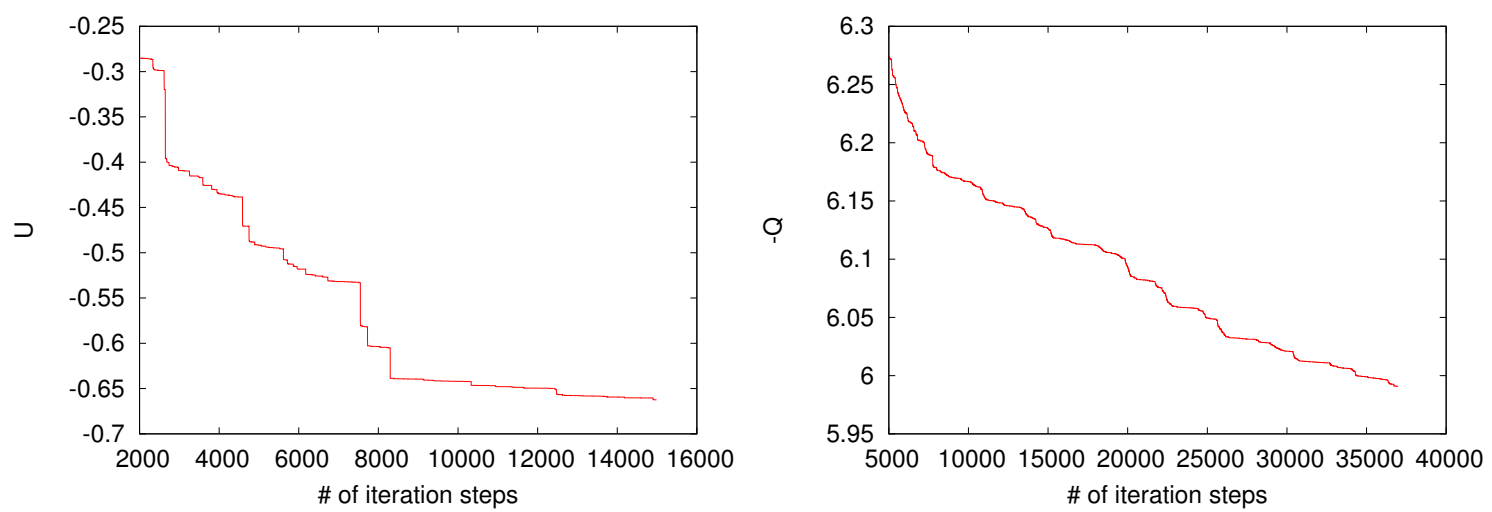

Figure 9. The behaviour of the objective functions $U$ and $-\mathrm{Q}$ (eq. (1.11) and (1.15)) as a function of the iteration steps in the $N_{\text {data }}$ (left) and $N_{\omega}$ (right) dimensional spaces.

\section{A MEM details}

\section{A.1 Numerical implementation}

The statistics currently feasible for dynamical Wilson-fermion calculations are very small compared to quenched simulations, meaning additional care has to be taken in the MEM analysis. We mention here the difficulties, that were not stressed earlier in the literature, summarizing our experience.

First of all, for realistic data double precision is not enough to make a reliable reconstruction of the SFs. Even in the case of the algorithm of ref. [23], which does not involve a Singular Value Decomposition, the Hessian matrix of the function $U$ has a big condition number, and arbitrary precision arithmetics is needed to find the correct minimum. Our implementation uses the Levenberg-Marquardt (or optionally the LBFGS) algorithm in arbitrary precision, implemeted by help of the GNU Multiple Precision Library. Using too small precision will generally lead to not finding the correct minimum. In practice, we have witnessed that using smaller precision leads to broader peaks.

Also, even with the high precision one has to be very careful about the stopping criteria for the iteration. The general behaviour is that after a rapid decrease of the function U, further iterations hardly improve it, i.e. it looks like hitting a plateau. Then after quite a few iterations, it starts improving fast once again, creating a step like pattern. With such a behaviour, one has to choose a very strict stopping criteria.

If we work in the original $N_{\omega}$ dimensional space (here, we can use the LBFGS algorithm), and maximize $\mathrm{Q}$ instead, this situation is slighly better. In that space, the behaviour is not step-like, but an iteration takes much more time, and whatever method one chooses, it takes lots of iterations to find the minimum, making the analysis computationally costly. This behaviour is illustrated on figure 9 .

In the end, we decided to follow the following procedure: minimize $U$ in the $N_{\text {data }}$ dimensional space, then after the iteration stops switch to the $N_{\omega}$ dimensional space and do some iterations to check if we found the true minimum, or not. 


\section{A.2 Error analysis}

We don't carry out an error analysis of the full spectral function, since with the current statistics that would give huge errors. Instead, we only give errors to some physically intersting quantities related to the spectral function, that are more stable.

The statistical error analysis is done with the usual jackknife method. For the sake of reducing computational cost, the statistical error estimate was only carried out on a given set of the parameters of the reconstruction algorithm. The systematic error analysis is carried out by varying the parameters of the reconstruction algorithm. Namely:

- the discretization of the frequency variable $\Delta \omega$

- the upper cut-off on the integral (1.6), $\omega_{\max }=N_{\omega} \Delta \omega$

- the shape and normalization of the prior function.

A numerical check on the lattice data and mock data analysis show that as long as $\Delta \omega$ is sufficiently small to resolve the peaks and $N_{\omega}$ is such that $\omega_{\max }$ is sufficiently big, which means somewhere around 5 inverse lattice spacings, the results are not affected by the choice of the first two parameters. In this analysis we used $a \omega_{\max }=5$ and $a \Delta \omega=0.005$. The effect of the prior function however, can not be neglected. For the systematical error analysis we used 3 different shapes:

- $m_{0} / a^{2}$, motivated by the philoshopy of "we know nothing".

- $m_{0} \omega^{2}$, motivated by continuum perturbation theory. We must stress however, that there is no reason to think that such an asymptotic behaviour can actually be seen on the lattice. In fact, analytical calculations of the SFs with free Wilson quarks show a different behaviour. (See [55] and [56]).

- $a^{-3} /\left(m_{0} / a+\omega\right)$, a theoretically unmotivated form. By using such a prior, we try to restrict the reconstructed shapes to the ones that are actually dictated by the lattice data.

- $m_{0} \omega / a$, motivated by the Kubo formula (1.10). This is the only prior function in the study that allows for a finite, nonzero diffusion constant, the others would imply either 0 , or infinity. We have only used this prior in the vector channel, where the analysis with the other prior functions suggested a transport peak.

In all cases, $a$ is the lattice spacing, and $m_{0}$ was varied between 10.0,1.0,0.1 and 0.01 to estimate the systematic errors. The systematic error was taken to be between the $17 \%$ and $83 \%$ percentiles of the sorted reconstructed parameters. The final errorbars on the plots include both systematic and statistical errors.

We mention, that it has been suggested (see e.g. [34]), that the free Wilson fermion SFs should be used as the prior information, we see no reason to do this however, since the free results have $\mathcal{O}(1)$ corrections in lattice perturbation theory, and treat the prior as a source of uncertainty instead. 
Open Access. This article is distributed under the terms of the Creative Commons Attribution License (CC-BY 4.0), which permits any use, distribution and reproduction in any medium, provided the original author(s) and source are credited.

\section{References}

[1] D.J. Schwarz, The first second of the universe, Annalen Phys. 12 (2003) 220 [astro-ph/0303574] [INSPIRE].

[2] Y. Aoki, G. Endrodi, Z. Fodor, S.D. Katz and K.K. Szabo, The order of the quantum chromodynamics transition predicted by the standard model of particle physics, Nature 443 (2006) 675 [hep-lat/0611014] [INSPIRE].

[3] Y. Aoki, Z. Fodor, S.D. Katz and K.K. Szabo, The equation of state in lattice QCD: With physical quark masses towards the continuum limit, JHEP 01 (2006) 089 [hep-lat/0510084] [INSPIRE].

[4] Y. Aoki et al., The QCD transition temperature: results with physical masses in the continuum limit II., JHEP 06 (2009) 088 [arXiv:0903.4155] [INSPIRE].

[5] S. Borsányi et al., The QCD equation of state with dynamical quarks, JHEP 11 (2010) 077 [arXiv: 1007.2580] [INSPIRE].

[6] A. Bazavov et al., The chiral and deconfinement aspects of the QCD transition, Phys. Rev. D 85 (2012) 054503 [arXiv:1111.1710] [INSPIRE].

[7] S. Borsányi, Z. Fodor, S.D. Katz, S. Krieg, C. Ratti and K. Szabó, Fluctuations of conserved charges at finite temperature from lattice QCD, JHEP 01 (2012) 138 [arXiv:1112.4416] [INSPIRE].

[8] НотQCD collaboration, A. Bazavov et al., Fluctuations and Correlations of net baryon number, electric charge and strangeness: A comparison of lattice QCD results with the hadron resonance gas model, Phys. Rev. D 86 (2012) 034509 [arXiv: 1203. 0784] [INSPIRE].

[9] T. Umeda et al., Fixed Scale Approach to Equation of State in Lattice QCD, Phys. Rev. D 79 (2009) 051501 [arXiv: 0809. 2842] [INSPIRE].

[10] WHOT-QCD collaboration, S. Ejiri et al., Equation of State and Heavy-Quark Free Energy at Finite Temperature and Density in Two Flavor Lattice QCD with Wilson Quark Action, Phys. Rev. D 82 (2010) 014508 [arXiv:0909.2121] [INSPIRE].

[11] WHOT-QCD collaboration, Y. Maezawa et al., Free energies of heavy quarks in full-QCD lattice simulations with Wilson-type quark action, Nucl. Phys. A 830 (2009) 247C-250C [arXiv:0907.4203] [INSPIRE].

[12] V.G. Bornyakov et al., Probing the finite temperature phase transition with $N(f)=2$ nonperturbatively improved Wilson fermions, Phys. Rev. D 82 (2010) 014504 [arXiv:0910.2392] [INSPIRE].

[13] WHOT-QCD collaboration, T. Umeda et al., EOS in 2+1 flavor QCD with improved Wilson quarks by the fixed-scale approach, PoS (Lattice 2010) 218 [arXiv:1011.2548] [INSPIRE].

[14] Y. Maezawa et al., Application of fixed scale approach to static quark free energies in quenched and 2+1 flavor lattice QCD with improved Wilson quark action, Prog. Theor. Phys. 128 (2012) 955 [arXiv:1112.2756] [INSPIRE]. 
[15] WHOT-QCD collaboration, T. Umeda et al., Equation of state in 2+1 flavor QCD with improved Wilson quarks by the fixed scale approach, Phys. Rev. D 85 (2012) 094508 [arXiv: 1202.4719] [INSPIRE].

[16] S. Borsányi et al., QCD thermodynamics with continuum extrapolated Wilson fermions I, JHEP 08 (2012) 126 [arXiv:1205.0440] [INSPIRE].

[17] S. Dürr et al., Ab-Initio Determination of Light Hadron Masses, Science 322 (2008) 1224 [arXiv:0906.3599] [INSPIRE].

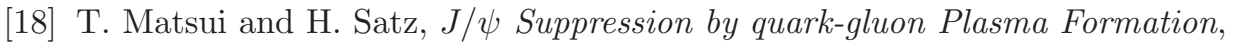
Phys. Lett. B 178 (1986) 416 [INSPIRE].

[19] M. Le Bellac, Thermal Field Theory, Cambridge University Press, (1996).

[20] M. Asakawa, T. Hatsuda and Y. Nakahara, Maximum entropy analysis of the spectral functions in lattice QCD, Prog. Part. Nucl. Phys. 46 (2001) 459 [hep-lat/0011040] [INSPIRE].

[21] G. Aarts and J.M. Martinez Resco, Transport coefficients, spectral functions and the lattice, JHEP 04 (2002) 053 [hep-ph/0203177] [INSPIRE].

[22] R.K. Bryan, Maximum-entropy analysis of oversampled data problems, Eur. Biophys J. 18 (1990) 165.

[23] A. Jakovác, P. Petreczky, K. Petrov and A. Velytsky, Quarkonium correlators and spectral functions at zero and finite temperature, Phys. Rev. D 75 (2007) 014506 [hep-lat/0611017] [INSPIRE].

[24] T. Umeda, A Constant contribution in meson correlators at finite temperature, Phys. Rev. D 75 (2007) 094502 [hep-lat/0701005] [INSPIRE].

[25] A. Rothkopf, Improved Maximum Entropy Analysis with an Extended Search Space, J. Comput. Phys. 238 (2013) 106 [arXiv:1110.6285] [InSPIRE].

[26] G. Aarts, C. Allton, J. Foley, S. Hands and S. Kim, Spectral functions at small energies and the electrical conductivity in hot, quenched lattice QCD, Phys. Rev. Lett. 99 (2007) 022002 [hep-lat/0703008] [INSPIRE].

[27] J. Engels and O. Vogt, Longitudinal and transverse spectral functions in the three-dimensional O(4) model, Nucl. Phys. B 832 (2010) 538 [arXiv:0911.1939] [InSPIRE].

[28] T. Umeda, K. Nomura and H. Matsufuru, Charmonium at finite temperature in quenched lattice QCD, Eur. Phys. J. C 39S1 (2005) 9 [hep-lat/0211003] [INSPIRE].

[29] M. Asakawa and T. Hatsuda, $J / \psi$ and $\eta(c)$ in the deconfined plasma from lattice QCD, Phys. Rev. Lett. 92 (2004) 012001 [hep-lat/0308034] [INSPIRE].

[30] G. Aarts, C. Allton, M.B. Oktay, M. Peardon and J.-I. Skullerud, Charmonium at high temperature in two-flavor QCD, Phys. Rev. D 76 (2007) 094513 [arXiv:0705.2198] [INSPIRE].

[31] A. Kelly, J.-I. Skullerud, C. Allton, D. Mehta and M.B. Oktay, Spectral functions of charmonium from 2 flavour anisotropic lattice data, arXiv:1312.0791 [INSPIRE].

[32] H. Iida, T. Doi, N. Ishii, H. Suganuma and K. Tsumura, Charmonium properties in deconfinement phase in anisotropic lattice QCD, Phys. Rev. D 74 (2006) 074502 [hep-lat/0602008] [INSPIRE]. 
[33] WHOT-QCD collaboration, H. Ohno et al., Charmonium spectral functions with the variational method in zero and finite temperature lattice QCD,

Phys. Rev. D 84 (2011) 094504 [arXiv:1104.3384] [INSPIRE].

[34] H.T. Ding, A. Francis, O. Kaczmarek, F. Karsch, H. Satz and W. Soeldner, Charmonium properties in hot quenched lattice QCD, Phys. Rev. D 86 (2012) 014509 [arXiv:1204.4945] [INSPIRE].

[35] G. Aarts et al., Charmonium spectral functions in two-flavour QCD, Nucl. Phys. A 785 (2007) 198 [hep-lat/0608009] [InSPIRE].

[36] A. Amato, G. Aarts, C. Allton, P. Giudice, S. Hands J.-I. Skullerud, Electrical conductivity of the quark-gluon plasma across the deconfinement transition, Phys. Rev. Lett. 111 (2013) 172001 [arXiv:1307.6763] [INSPIRE].

[37] G. Aarts, C. Allton, S. Kim, M.P. Lombardo, S.M. Ryan J.-I. Skullerud, Melting of P wave bottomonium states in the quark-gluon plasma from lattice NRQCD, JHEP 12 (2013) 064 [arXiv: 1310.5467] [INSPIRE].

[38] K. Symanzik, Continuum Limit and Improved Action in Lattice Theories. 1. Principles and $\Phi^{4}$ Theory, Nucl. Phys. B 226 (1983) 187 [InSPIRE].

[39] M. Lüscher and P. Weisz, On-Shell Improved Lattice Gauge Theories, Commun. Math. Phys. 97 (1985) 59 [Erratum ibid. 98 (1985) 433] [INSPIRE].

[40] B. Sheikholeslami and R. Wohlert, Improved Continuum Limit Lattice Action for QCD with Wilson Fermions, Nucl. Phys. B 259 (1985) 572 [inSPIRE].

[41] C. Morningstar and M.J. Peardon, Analytic smearing of SU(3) link variables in lattice QCD, Phys. Rev. D 69 (2004) 054501 [hep-lat/0311018] [INSPIRE].

[42] R. Hoffmann, A. Hasenfratz and S. Schaefer, Non-perturbative improvement of nHYP smeared Wilson fermions, PoS(LATTICE 2007) 104 [arXiv:0710.0471] [INSPIRE].

[43] S. Capitani, S. Dürr and C. Hölbling, Rationale for UV-filtered clover fermions, JHEP 11 (2006) 028 [hep-lat/0607006] [INSPIRE].

[44] S. Dürr et al., Scaling study of dynamical smeared-link clover fermions, Phys. Rev. D 79 (2009) 014501 [arXiv:0802.2706] [INSPIRE].

[45] S. Duane, A.D. Kennedy, B.J. Pendleton and D. Roweth, Hybrid Monte Carlo, Phys. Lett. B 195 (1987) 216 [INSPIRE].

[46] M.A. Clark and A.D. Kennedy, Accelerating dynamical fermion computations using the rational hybrid Monte Carlo (RHMC) algorithm with multiple pseudofermion fields, Phys. Rev. Lett. 98 (2007) 051601 [hep-lat/0608015] [INSPIRE].

[47] J.C. Sexton and D.H. Weingarten, Hamiltonian evolution for the hybrid Monte Carlo algorithm, Nucl. Phys. B 380 (1992) 665 [INSPIRE].

[48] T. Takaishi and P. de Forcrand, Testing and tuning new symplectic integrators for hybrid Monte Carlo algorithm in lattice QCD, Phys. Rev. E 73 (2006) 036706 [hep-lat/0505020] [INSPIRE].

[49] T.A. DeGrand, A Conditioning Technique for Matrix Inversion for Wilson Fermions, Comput. Phys. Commun. 52 (1988) 161 [inSPIRE].

[50] C.T.H. Davies et al., Precise Charm to Strange Mass Ratio and Light Quark Masses from Full Lattice QCD, Phys. Rev. Lett. 104 (2010) 132003 [arXiv:0910.3102] [InSPIRE]. 
[51] S. Dürr et al., Lattice $Q C D$ at the physical point: light quark masses, Phys. Lett. B 701 (2011) 265 [arXiv: 1011.2403] [INSPIRE].

[52] S. Dürr et al., Lattice QCD at the physical point: Simulation and analysis details, JHEP 08 (2011) 148 [arXiv: 1011.2711] [INSPIRE].

[53] S. Borsányi et al., Anisotropy tuning with the Wilson flow, arXiv:1205.0781 [INSPIRE].

[54] G.I. Egri et al., Lattice QCD as a video game, Comput. Phys. Commun. 177 (2007) 631 [hep-lat/0611022] [INSPIRE].

[55] F. Karsch, E. Laermann, P. Petreczky and S. Stickan, Infinite temperature limit of meson spectral functions calculated on the lattice, Phys. Rev. D 68 (2003) 014504 [hep-lat/0303017] [INSPIRE].

[56] G. Aarts and J.M. Martinez Resco, Continuum and lattice meson spectral functions at nonzero momentum and high temperature, Nucl. Phys. B 726 (2005) 93 [hep-lat/0507004] [INSPIRE]. 